\title{
Unraveling the Immune Response in Severe COVID-19
}

\author{
Lucie Rodriguez $^{1} \cdot$ Petter Brodin ${ }^{1,2}$ (D)
}

Received: 15 August 2020 / Accepted: 18 August 2020 / Published online: 22 August 2020

(C) Springer Science+Business Media, LLC, part of Springer Nature 2020

An outbreak of coronavirus disease 2019 (COVID-19) emerged in China's Hubei province and spread rapidly across the globe leading to millions of cases worldwide. The causative agent, severe acute respiratory syndrome coronavirus 2 (SARS-CoV-2), a novel enveloped RNA-virus of the family of beta-coronavirus, was quickly identified and sequences distributed, initiating a race to develop vaccines and therapeutics that is unprecedented in history. A striking feature of the COVID-19 disease is the wide range of disease severity across different infected individuals. The SARS-CoV-2 infection is almost exclusively mild in children [1], while older individuals, as well as those with specific preexisting illnesses, are more likely to develop severe disease and require intensive care [2]. One exception from this general rule is the postinfectious condition termed multisystem inflammatory syndrome (MIS-C) affecting children with COVID-19, a life-threatening syndrome developing 2-6 weeks after the acute SARS-CoV-2 infection [3]. The immunology of this condition is currently under intense investigation [4]. Overall, there is an urgent need to better understand the determinants of this difference in COVID-19 disease presentation, the underlying genetic and functional differences between mild and severe disease patients [5], and ideally also identification of useful biomarkers that can be used to monitor disease progression and distinguish productive from deleterious immune responses.

SARS-CoV-2 infection and the immune response to the virus can be divided into two phases. In the first phase, the virus replicates in the respiratory epithelium and alveoli and the principal antiviral response mechanism is the type-I IFN response aimed at limiting viral replication. If this is

Petter Brodin

petter.brodin@ki.se

1 Science for life Laboratory, Department of Women's and Children Health, Karolinska Institutet, Stockholm, Sweden

2 Pediatric Rheumatology, Karolinska University Hospital, Stockholm, Sweden insufficient at limiting the infection, there is a second phase in patients with more severe disease course, in which cell death and local inflammation triggers the induction of additional cytokines and chemokines that draw additional immune cells into the lung. At the same time, danger signals and alarmins like HMGB1 are released and possibly trigger a self-enhancing inflammatory response giving rise to acute respiratory distress syndrome (ARDS) and clinical deterioration. There has been much discussion around the specifics of this second phase dysregulated immune response in severe COVID-19 but the actual data remain scarce [6]. We know that lymphopenia is a hallmark of severe disease and the degree of lymphopenia, and neutrophil-to-lymphocyte ratio correlates with disease severity [7]. The same is true for plasma levels of lactate dehydrogenase, a marker of inflammasome activation and pyroptosis [8] which correlate with disease severity.

In this issue of the Journal of Clinical Immunology, four papers add substantial additional information to our current understanding of the immune response and immunopathology in severe COVID-19. Mahmoudi et al. review this rapidly evolving field with a particular focus on the role of exhausted effector T cells and NK-cells, as well as impaired regulatory T cell populations in severe COVID-19 and the cytokine storm associated with this condition.

Given the dynamic nature of immune responses to infections and particularly when studying samples from human patients with an unknown time from initial viral exposure, longitudinal monitoring is critical in order to reveal the coordinated changes and sequences of events. Several recent studies have reported longitudinal monitoring of patients with COVID-19 [9-11] with up to 14 longitudinal samples collected from individual patients [11]. Adding to these studies in the current issue, Moratto et al. report dynamic changes in immune cell composition over time in 36 patients with COVID-19 in Brescia, Italy, a city severely affected by the pandemic during the spring of 2020. The authors performed detailed flow cytometry analyses in these patents. Eight patients had moderate disease, 28 had severe COVID-19, and 22 
of these recovered, while 6 of the severe cases died. Remarkably, Moratto et al. find that immune cell subsets differ among these groups already at first admission, suggesting different disease trajectories manifested early after admission, in some cases before disease severity is evident from the clinical evaluation. The differences involve the severity of lymphopenia but also specific reductions in gdT-cell counts and a skewing of abT-cells towards more exhausted phenotypes. These interesting observations corroborate previous studies and also add some novel aspects of the immune dysregulation and immunopathology in adults with critical COVID-19. The data also indicate the potential of these immune system measurements to be predictive of disease severity in individual patients.

Along the same lines, Luo et al. report in this same issue a large study of 739 COVID-19 patients treated at Tongjio Hospital, Wuhan, China. These authors used lymphocyte subset analyses, plasma cytokine levels to train a predictive model with both sensitivity and specificity at $90 \%$, although the testing of the model in an unrelated cohort remains to be done. Together, these reports indicate that prediction of disease trajectory might be possible early upon admission to hospital, which will be incredibly valuable in any healthcare system struggling to prioritize limited resources to the patients with the greatest need.

In human immunology, detailed analyses of individual patients with unique clinical presentation have proven incredibly informative and driven much of the characterization of the $~$ 400 primary immunodeficiency disorders known to date [12]. Bouadma et al. report in this issue a careful examination of the immune response in a single patient who succumbed to COVID-19, and this analysis corroborates several of the immune features discussed above from population studies. For example, this patient had a skewing of $\mathrm{T}$ cell subsets towards exhausted abT-cells, as well as a remarkable expansion of gdT-cells during deterioration, while monocytes largely disappeared from circulation, supposedly due to redistribution and homing to the lung during ARDS.

In their unique way, each of these new papers adds additional layers to our understanding of the immune response and dynamical changes in severe COVID-19. They highlight the power and potential of longitudinal and systems-level analyzes in human immunology in order to reveal co-regulated cells and proteins over time[13] and ideally even predict outcome in individual patients opening up for more personalized management of COVID-19 patients in the future.

\section{Compliance with Ethical Standards}

Conflict of Interest The authors declare that they have no conflicts of interests.

\section{References}

1. Brodin P. Why is COVID-19 so mild in children? Acta Paediatr. 2020.

2. Guan W-J, Ni Z-Y, Hu Y, Liang W-H, Ou C-Q, He J-X, et al. Clinical characteristics of coronavirus disease 2019 in China. $\mathrm{N}$ Engl J Med. 2020;382:1708-20.

3. Whittaker E, Bamford A, Kenny J, Kaforou M, Jones CE, Shah P, et al. Clinical characteristics of 58 children with a pediatric inflammatory multisystem syndrome temporally associated with SARSCoV-2. JAsMA. 2020;324.

4. Consiglio CR, Cotugno N, Sardh F, Pou C, Amodio D, Zicari S, et al. The immunology of multisystem inflammatory syndrome in children with COVID-19. MedRxiv [Internet]. 2020; Available from: https://doi.org/10.1101/2020.07.08.20148353

5. Casanova J-L, Su HC, Effort CHG. A global effort to define the human genetics of protective immunity to SARS-CoV-2 infection. Cell. 2020;181:1194-9.

6. Tay MZ, Poh CM, Rénia L, MacAry PA, Ng LFP. The trinity of COVID-19: immunity, inflammation and intervention. Nat Rev Immunol. 2020;20:363-74.

7. Lagunas-Rangel FA. Neutrophil-to-lymphocyte ratio and lymphocyte-to-C-reactive protein ratio in patients with severe coronavirus disease 2019 (COVID-19): a meta-analysis. J Med Virol. 2020.

8. Evavold CL, Ruan J, Tan Y, Xia S, Wu H, Kagan JC. The poreforming protein gasdermin D regulates interleukin-1 secretion from living macrophages. Immunity. 2018;48:35-44.e6.

9. Lucas C, Wong P, Klein J, Castro TBR, Silva J, Sundaram M, et al. Longitudinal analyses reveal immunological misfiring in severe COVID-19. Nature. 2020:1-9.

10. Mathew D, Giles JR, Baxter AE, Greenplate AR, Wu JE, Alanio C, et al. Deep immune profiling of COVID-19 patients reveals patient heterogeneity and distinct immunotypes with implications for therapeutic interventions. Biorxiv. 2020;2020.05.20.106401.

11. Rodriguez L, Pekkarinen PT, Lakshmikanth T, Tan Z, Consiglio $\mathrm{CR}$, Pou C, et al. Systems-level immunomonitoring from acute to recovery phase of severe COVID-19. Cell Rep Med. 2020;100078.

12. Notarangelo LD, Bacchetta R, Casanova JL, Su HC. Human inborn errors of immunity: an expanding universe. Sci Immunol. 2020;5: eabb1662.

13. Lakshmikanth T, Muhammad SA, Olin A, Chen Y, Mikes J, Fagerberg L, et al. Human immune system variation during 1 year. Cell Rep. 2020;32:107923.

Publisher's Note Springer Nature remains neutral with regard to jurisdictional claims in published maps and institutional affiliations. 